University of Warwick institutional repository

This paper is made available online in accordance with

publisher policies. Please scroll down to view the document

itself. Please refer to the repository record for this item and our

policy information available from the repository home page for further information.

To see the final version of this paper please visit the publisher's website. Access to the published version may require a subscription.

Author(s): Marilyn Hunt; Ann Barnes

Article Title: A potential library for primary MFL pedagogy: the case of

Young Pathfinders

Year of publication: 2006

Link to published version: http://dx.doi.org/10.1080/09571730685200131

Publisher statement: None. 


\section{A Potential Library for Primary MFL pedagogy: the case of Young Pathfinders}

Marilyn Hunt and Ann Barnes, University of Warwick Institute of Education

\section{Introduction}

As readers of this journal will know very well, 2010 will see all KS2 (ages 711) pupils in England entitled to learn a modern foreign language in normal curriculum time. This development of the commitment to primary language learning should provide an excellent opportunity and experience for pupils, whilst at the same time requiring some radical changes for many teachers, schools and much of the wider language learning community.

Recent research has indicated general trends suggesting an increase in primary languages already, in anticipation of this development and even beforehand. One of the most recent studies indicates that $43 \%$ of primary children currently learn a foreign language at KS2, either in class or as an extra-curricular activity, although the extent of this learning varies considerably (Driscoll, Jones and Macrory, 2004). It has also been suggested (Muijs et al, 2005) that there are certain aspects of the process that will be particularly demanding if the challenge of providing this entitlement are to be met.

Key issues for this development include:

- Phase specific pedagogy for secondary specialist teachers

- Subject specific pedagogy for primary non-specialist teachers

- Linguistic knowledge, competence and competence, particularly for primary non-specialist teachers

- The challenge of ensuring continuity and progression for an effective transition for learners from KS2 to KS3

How these key issues are addressed will vary according to the needs of the people involved and the resources available. In addition to face to face support and the increasing number of early language learning networks, one possible source of support for teachers involved in primary foreign languages is through published methodological material. There is now a wide range of this type of material available and the offer is growing continually. This article focuses on one particular series, published by CILT.

\section{The Young Pathfinder series: overview}

First published in 1995 and added to regularly since then, the CILT series Young Pathfinders provides a valuable source of help, support and advice to the specialist and non-specialist alike in the primary languages arena. The series taken as a whole goes a substantial way to addressing the key issues outlined above. With any resources for primary foreign languages, it is vital to consider both specialists and non-specialists, working in a range of different contexts and who need varying amounts of linguistic and subject specific methodological advice. For non-specialist teachers of foreign languages in a primary setting, such resources are crucial, as there may be only one person in the school working within foreign language teaching and learning. As recent research has shown (Muijs et al, 2005), teachers are particularly keen 
to receive help with schemes of work, confidence in the subject specific methodology, and confidence and competence linguistically. Resources such as those reviewed here can help greatly with many of these issues. For secondary languages specialists, important facets of the primary context emerge clearly from this series, and would therefore help such teachers adapt their approaches to suit the learners.

A great advantage of the Young Pathfinder series is that each volume is quick and easy to read, and written in simple, concise language often with bullet points outlining practical content and useful ideas for the classroom. This makes them easily accessible and easy to return to for reference, without demanding a great deal of time. They are also therefore ideal for sharing around within a department, school or school cluster and can be used as preparatory reading for a training session, for example. They are written by experienced authors, trainers and practitioners and contain a number of very sound nuggets of methodological advice, incorporated in to the deceptively thin and straightforward volumes. All authors are clearly very well aware of the primary context, and the best examples address explicitly the possible concerns teachers may have. Each of the volumes forms one part towards a coherent whole; the books and themes are interrelated and cross-referenced. Many contain extra advice and ideas on a theme which is covered more specifically in a separate volume. Thus, there is little risk of teachers receiving confusing or mixed messages. Most of the volumes include to a greater or lesser extent songs, games and other activities and would, together, build up to a thorough, accessible library for teachers of foreign languages in a primary setting. We have chosen a number of key themes for this article, in order to review the series holistically ${ }^{1}$.

\section{Planning and managing primary foreign language learning}

As an introduction to the primary foreign languages context, teachers and curriculum managers could begin by consulting A flying start! Introducing early language learning (de Silva and Satchwell, 1995/2004), which is a fully revised version of Catching them young (Young Pathfinder 1) and provides up-to-date information about how the early language learning context has progressed, a detailed list of current sources of support and important developments in assessment and ways to help with primary secondary liaison. It presents a rationale for early language learning, outlines key aims and objectives, and provides advice for primary teachers about to embark on a new foreign language programme in terms of planning themes and topics and a scheme of work to include progression in linguistic and learning skills and grammar.

In some volumes in the series, e.g. Games and Fun Activities (Martin, 1995), where the focus is explicitly on activities and engagement, a 'coherent programme' is alluded to, but it is often this coherent programme which is the challenge: such as when to use these activities and in what context. Taken

This review article focuses solely on those books submitted for review: ${ }^{1}$ not all volumes in the series were available 
as a whole, however, the Young Pathfinders begin to piece together such a coherent programme, where activities are not merely used because they are fun, but because they serve a broader purpose in foreign language learning. Keep Talking. Teaching in the target language (Satchwell, 1998), for example, acknowledges the wider learning that takes place in the primary classroom, beyond the language itself and contains very helpful advice on practical matters and methodology. One suggestion might be that this book should be read first by the non-specialist, as it contains some fundamental messages. It would also be possible to begin to build up embryonic schemes of work from some parts of the book.

One of the issues of languages entitlement throughout KS2 is the ability to staff it with competent and confident teachers in the foreign language. One solution to this challenge as outlined in the National Languages Strategy (2002) is the use of native speakers. In Working together: Native speaker assistants in the primary school (2004) Martin and Farren show how native speaker assistants can make a vital contribution to work in the primary school, not only in early foreign language learning but also in other ways too through their cultural input and involvement in the everyday life of school. The authors of this volume provide considerable information on practical organisational issues such as how to find a native speaker and different models for organizing and timetabling native speakers, all well illustrated with detailed examples and useful tips, as well as quotations from heads, teachers and native speakers from real experiences. However, Martin and Farren also make it clear how vital the planning and preparation process is to ensure a successful placement, outlining how local authorities, HEls or secondary schools might help primary schools prepare to host a native speaker assistant. They suggest approaches to making important practical arrangements before a native speaker's arrival in the primary school, for example, appointing a mentor and clarifying expectations, and there are useful checklists in the volume both for the mentor and native speaker prior to departure. The authors provide details of a suggested induction programme and some very helpful advice on how to ensure both the native speaker and the school derive the most benefit through shared planning, and other supports such as monitoring and reviewing progress.

\section{Engaging young learners in foreign language learning}

A number of volumes in the series focus specifically on the issue of engagement and motivation, whilst many others incorporate such approaches in to their particular focus. Suggestions of activities included in this type of resource can then act as springboards for teachers to stimulate other activities, outcomes, and implementation, arising from teachers' own context, experience and imagination. Games and Fun Activities (Martin, 1995) and Let's join in! Rhymes, Poems and Songs (Martin and Cheater, 1998) are directly aimed at enthusing young learners whilst enabling them simultaneously to make progress in the language. In spite of its early publication date (and despite showing its age with references to games at the board using white and coloured chalk), the first of these still includes some good practical examples of games to motivate early learners. It contains 
suggestions for games during lesson time or those which could be incorporated into the daily routine of the class, or alternatively outdoors on the field or in the playground. Helpful step by step instructions are provided. Games include tried and tested favourites such as hangman, noughts and crosses, guessing and memory games and useful advice about card games that can be adapted to use the language in a real context such as Happy Families and dominoes. Many of these could also be used (or adapted for use) in key stage 3 or beyond. The book also includes craft activities and an example of cooking and games/action rhymes or songs with music, thus creating some inevitable overlap with Let's join in! Rhymes, Poems and Songs (Martin and Cheater, 1998). The latter book contains rhymes, songs and poems aimed at specialist and non-specialist teachers of French and German. Part 1, which focuses on examples which are suitable for learners from pre-school through KS1 to the early stages of KS2, provides suggestions for a range of speaking, clapping and action rhymes. The emphasis is on frequency of exposure using short bursts of language which can become part of the daily routine of the class. Examples of singing rhymes are also included with suggestions as to how to create new versions, for example for counting, using familiar tunes. For non-primary teachers, reminders of such finger rhymes and action songs are invaluable, and for those to whom the concept is familiar the linguistic examples are helpful. Another way of engaging learners is provided in First steps to reading and writing, where Skarbek (1998) suggests some useful ideas for encouraging creative writing using acrostic poetry or a structure or framework, as well as more open-ended tasks. Similarly, Are you sitting comfortably? Telling stories to young language learners (Tierney and Dobson, 1995) is a very practical guide to using stories with young learners with some crossover with other volumes in the Young Pathfinders series.

\section{Foreign language methodology and pedagogy}

The authors of The Literacy Link (Cheater and Farren, 2001) aim to show teachers the beginnings of methodology which can integrate a modern foreign language into the language and literacy development of young learners. Suggestions are divided into word, sentence and text level to reflect the National Literacy Strategy. At word level, using French, German and Spanish examples and comparing with English, the authors focus on games and activities for recognising and discriminating sounds, sequencing and sorting by initial letter and developing awareness of the critical features of words, for example the number of syllables, length of the word, and combination of vowels and consonants. As is usual in this series, the authors provide a careful rationale for the premise explored in the book, and clear guidelines and advice to realise the ideas in the classroom. These rationales are invaluable in disseminating methodological advice in a coherent context and framework. Martin and Cheater (1998:63) for example emphasise the importance of rhymes and songs for language learning: '... rhymes and songs are vehicles which can permit learners to shape their mouths and utter foreign sounds when they might otherwise not have wished to do so. Their use can build confidence and self-esteem..... This type of rationale helps embed rationalised methodological approaches in to the teaching and learning, rather 
than presenting a series of unconnected activities. In addition to clear introductions and rationales, volumes in this series achieve a clarity of advice on implementation: vital for a practitioner who may be somewhat lacking in confidence and experience. All the ideas in Let's join in! Rhymes, Poems and Songs (Martin and Cheater, 1998), for example, are practical and described in detail to help teachers consider rhymes, songs or activities which could be presented using visuals and realia and this is especially helpful for non-specialists. Some rhymes are provided to fit into particular themes, for example, weather, animals, or to accompany popular stories such as 'The Hungry Caterpillar'. Many examples of songs are provided using familiar tunes as well as reference to published audio cassettes and video.

The four language skills (listening, speaking, reading and writing) are dealt with in a variety of ways through the series. In First steps to reading and writing, Skarbek (1998:2) states that 'For too long we have concentrated disproportionately on listening and speaking, to the detriment of the other two skills'. Clear methodological steps are again provided in this volume. The book begins by outlining ways of exposing children to the written word through labels in the classroom, weather and calendar charts and authentic posters. It goes on then to describe ways of connecting the spoken word to its written form, by gradually removing visual links, building on familiar, well-known songs or rhymes using gap-fill. Story-telling is also a focus: ideas are provided for appropriate stories which encourage curiosity and use easily recognised and repeated patterns of language. One section concentrates on developing initial reading skills and contains some helpful ideas and word games. Particularly helpful is the inclusion of strategies for reading and writing.

As mentioned above, there is some overlap across these Young Pathfinders as Keep Talking: Teaching in the target language (Satchwell, 1997) comments on all four skill areas not just speaking, and includes songs, rhymes and games. This is to be welcomed: language learning and methodological approaches do not fit neatly in to distinct categories, but should form part of a coherent whole. For example, after providing a rationale for storytelling and describing the qualities needed of both the story and the storyteller, Tierney and Dobson in Are you sitting comfortably? Telling stories to young language learners (1995) helpfully outline possible ways to lead in to a story which involve presentation of vocabulary with a variety of visuals and a range of games which could be played to practise this new vocabulary. They provide ideas of how to tell the story for the first time and how to extend it subsequently by developing the language further, for example, matching pictures to text, sequencing text, acting out the story through mime or indeed memorising or creating their own script. The authors present a list of suggested stories which have been used successfully with language learners and supply details of how one specific story could be used in class. A flying start! Introducing early language learning (de Silva and Satchwell, 1995/2004) reiterates methodology which has proved successful in the primary phase, for example, games, songs, rhymes, drama and storytelling, many of which overlap with ideas encountered in other Young Pathfinders. The benefits of storytelling are apparent in a number of Young Pathfinders 
and in A world of languages: Developing children's love of languages (2004), Datta and Pomphrey supply convincing arguments for using stories from diverse language and cultural backgrounds. They show how stories can expand children's confidence and thinking as users or learners of language and how they develop a deeper knowledge and understanding about language, culture and identity. They demonstrate activities from stories and how stories can bring humour, fun, imagination and collaboration to the languages classroom.

Languages for primary aged learners should of course move beyond straightforward repetition and vocabulary learning. Grammar is the tool in the language learner's box which enables new language to be generated. In Grammar is fun! (Biriotti, 1999), the author starts with an interesting way of introducing the notion of gender by encouraging learners to memorise certain words of a particular gender in a story told in English where the teacher reinforces the words in the target language. The author goes on to explain how the notion of agreements of adjectives can be introduced in the same way and has some good ideas for practising, for example being shrunk by a space ray so that everything is huge, or the world turning red or green as well as a range of good practical ideas and interactive games, songs, mimes and activities for reinforcing grammatical rules and patterns. Detailed ideas for introducing, practising and reinforcing other grammatical concepts such as plurals, prepositions, verbs and adverbs are also presented, many of which are quite innovative. Biriotti clearly outlines the use of checklists, colour coded mnemonics and grids and schematic prompts, all interwoven in interactive games, to help learners assimilate the concept of different persons to build a firm foundation for understanding the mechanisms of verbs. In some cases the language level is quite ambitious and the ideas could equally be used in KS3.

\section{Linguistic competence and confidence for non-specialist teachers of young learners}

In Keep Talking. Teaching in the target language, Satchwell (1997) stresses the importance of sustaining target language in the classroom and warns 'Your pupils will only 'keep talking' in the target language if you do so!' This, of course, highlights the current challenge of teacher competence and confidence in the target language to satisfy the needs of pupil entitlement to a foreign language throughout KS2. Satchwell provides convincing arguments for using the target language, yet sensitively encourages the non-specialist to feel capable of doing so, giving reasons for the judicial use of English/mother tongue. It therefore addresses the linguistic confidence issue head-on, maintaining an encouraging tone and providing a very useful checklist outlining in exactly which aspects of language a teacher should be competent. The book is also realistic about the fact that teachers will make linguistic mistakes. Satchwell draws a distinction between teacher and pupil talk and provides helpful examples of teacher classroom instructions with visuals (and a detailed appendix for most circumstances) and a 'survival kit' of pupil classroom phrases as well as ways of encouraging pupils to use language actively through games, stories and the use of puppets. One chapter in $A$ 
flying start! Introducing early language learning (de Silva and Satchwell, 1995/2004) makes the case for the use of target language and provides valuable encouragement for the type of target language that can be expected from both primary teachers and pupils.

Most target language examples in Games and Fun Activities (Martin, 1995) are in French although there are some in both French and German and a useful glossary at the back containing both teacher and pupil language. The target language instructions (in French) could help the non-specialist, although the praise expressions mixed in French and German could be a little confusing and an explanation of the differing forms of the imperative near the beginning of the book would have perhaps been of benefit. Linguistic examples in First steps to reading and writing (Skarbek, 1998) and Keep Talking: Teaching in the target language (Satchwell, 1997)are all in French and German. In the latter volume, a glossary contains additional resources which Satchwell recommends throughout and the varying imperative forms are explained. The fact that Spanish is generally not exemplified in the series as a whole is somewhat disappointing, but understandable from a manageability perspective (although Spanish examples are included in The Literacy Link, Cheater and Farren, 2001). Suggestions for integrating the foreign language into aspects of the curriculum included in Making the link Relating languages to other work in the school Tierney and Hope (1998) such as Maths, Geography, Design and Technology, Music and Drama range from less complex linguistic ideas to those which would require greater confidence and competence linguistically on the part of the teacher using French as the medium for teaching aspects of the National Curriculum subjects and RE.

\section{$5 \quad$ Foreign languages and the wider primary curriculum}

One of the concerns of introducing a foreign language into the primary phase is the issue of time in an already packed curriculum. In Making the link Relating languages to other work in the school (1998), Tierney and Hope illustrate how the children's language experience can be linked to the other work going on in the classroom and how aspects of the primary curriculum can even be taught through the medium of the foreign language. They suggest ways that the foreign language can be part of the physical environment and be used for everyday classroom instructions throughout the day. The authors also develop different themes which could be used as a rich context for language work, for example, the village, or a cross-curricular theme such as healthy living and links to festivals. Learning aims and objectives, vocabulary and cross-referencing to the NC are included throughout.

Links to cross-curricular themes are more implicit in Let's join in! Rhymes, Poems and Songs (Martin and Cheater, 1998) e.g. literacy: making up songs using animal words of one or three syllables, developing awareness of the 'rhythm' of individual words. Part 1 of this volume ends with an example of bringing it all together to fit in with other primary themes and provides examples of how rhymes and songs can be reworked and fitted into a nativity play. Keep Talking. Teaching in the target language (Satchwell, 1997) also 
includes a chapter on 'Display', which whilst useful, is disappointingly lacking in suggesting how display might influence and encourage pupils to 'keep talking'!

The concept of linking a story to other aspects of the curriculum is successfully demonstrated in Are you sitting comfortably? Telling stories to young language learners (Tierney and Dobson, 1995) with a detailed example of extending the stories Dear Greenpeace and My friend whale in German to the theme of conservation. There are some good ideas for making a dictionary in The Literacy Link (Cheater and Farren, 2001), and dictionary projects which clearly link with literacy. This book also contains some excellent ideas for working at sentence level using flashcards to create sentences which can lead forward to reading and writing, and for working at text level where many of the activities include ways to differentiate. There is some overlap understandably with Grammar is fun, for example ways of introducing and practising nouns, adjectives, verbs and adverbs and with Are you sitting comfortably? when giving ideas for storytelling as a means of working with text, although this is extended to a range of ideas for re-creating stories.

Mind the gap! Improving transition between Key Stage 2 and 3 (Bevis and Gregory, 2005) includes photographs, examples of documentation and diagrams throughout which add considerably to its appeal and provide some very helpful insights in to primary practice, particularly for secondary specialists. As they state in A world of languages: Developing children's love of languages Datta and Pomphrey (2004:1) believe a multilingual approach to language development helps 'to deepen children's understanding of what language is, how it works and how it is used in different contexts, which in turn strengthens the teaching and learning of the National Literacy Strategy at text, word and sentence level.' They explore how to capitalise on the linguistic and cultural diversity learners encounter in their everyday lives and how to encourage children to talk about their personal experiences and perceptions of languages as a means of showing knowledge of language acquisition, awareness of other languages, thoughts about how to learn languages and links between language and identity and language and culture. One chapter focuses on managing multilingual activities in order to create positive attitudes towards language learning and investigates ways in which bilingual adults can contribute. Datta and Pomphrey also emphasise the importance of working with words, phrases and sentences in a variety of languages to reinforce the metalinguistic concepts featured in the National Literacy Strategy and show how skills and concepts are transferable between languages. The authors include some excellent examples of finding similarities between languages in order to strengthen children's knowledge and understanding about how language works. One activity is a cross-curricular example of teaching a science topic of sinking and floating in Hindi by a bilingual teacher. Multilingual activities provide excellent motivation and preparation for learning new languages in the future and can draw on the many languages present in many of our classrooms nowadays. As Datta and Pomphrey state: 'Sharing children's language histories, knowledge and skills is empowering for every child, and creates the inspiration and motivation to learn a new language.' 
Interactive activities, for example, drama, games, singing, investigations, cooking, stories and rhymes, which form a standard part of the primary curriculum, could equally be carried out in other languages as well as English and Datta and Pomphrey supply many exciting ideas and language examples which have been used successfully. In Working together: Native speaker assistants in the primary school (2004) Martin and Farren suggest possible topics to be exploited by a native speaker assistant, such as how to enhance intercultural understanding, cross-curricular links and the potential for special events.

\section{Progression and transition to KS3}

Mind the gap! Improving transition between Key Stage 2 and 3, (Bevis and Gregory, 2005) is a crucial text when considering this issue; an aspect of the primary languages development which could be a serious hindrance to successful implementation and continued sustainability. The importance of building effectively on pupils' achievements as they move into secondary school is clearly recognised. Indeed, one of the explicit objectives of the KS3 strategy is to improve progression across the key stages and this issue was discovered to be central in ensuring success and progression in a recent study (Muijs et al, 2005). An Ofsted report (2002) noted that continuity in the curriculum and progression in learning as pupils move from primary to secondary schools are longstanding weaknesses of the education system. If difficulties emerge in NC statutory core subjects (English, Mathematics and Science), it is easy to imagine the difficulties of transfer in MFL where pupils may come to a secondary school from a range of feeder primary schools where 'entitlement' allows variety in language provision in time allocation, teaching quality (both subject knowledge and pedagogic expertise), and indeed the particular language studied.

Mind the gap! Improving transition between Key Stage 2 and 3, thanks to its recent publication date, is refreshingly up-to-date with the current context, policy and documentation (KS2 Framework, etc), methodology and crosscurricular links. Bevis and Gregory outline the problems connected with diversity of provision and tackle this issue by helping to bridge the gap between the primary and secondary phases to establish and maintain continuity and progression. They suggest ways in which primary and secondary teachers can develop effective liaison and joint planning, exploring practical activities, joint events and methodology. 'It is only by meeting 'the other side' that teachers can begin to work together for the child's benefit'. They include a helpful example of an action plan from the NACELL best practice guide (2003) and sensitively acknowledge the potential lack of understanding between primary and secondary colleagues in the past and suggest ways to communicate between both phases to overcome this. The emphasis throughout is on ways of achieving continuity and progression through, for example, developing a shared scheme of work, transferring information about pupils' progress through the European Language Portfolio and using assessment, in particular the Languages Ladder National Recognition Scheme. Different ways to support pupils in the KS2/3 transfer are discussed, for example, top-up classes, as well as good ideas for bridging 
units, collaborative projects and detailed ideas for special events. One chapter focuses on how to organise a special event, with a useful checklist and practical considerations for anyone wishing to stage such an event and an example of one specific topic with plans for the workshops/activities throughout the day. 'For the primary and secondary teachers, this type of event provides an opportunity to observe different teaching styles and activities which can be copied, to work together with a shared goal, alongside pupils they have taught or will teach and even to assess their achievement informally' p.64 The final chapter considers how to cater for Year 7 mixedlevel groups and again provides some very good practical suggestions for maintaining motivation and achieving progression and continuity through effective differentiation. The implementation of such ideas will be crucial in secondary schools where MFL teachers in Year 7 will need to cater for a wide diversity of prior knowledge and skills.

Other volumes in the series also tackle this crucial issue. Skarbek (1998:1) in First steps to reading and writing points out sensibly that 'Pupils must feel that the work they are doing is appropriate to their needs and they should not be presented with the same language and activities year after year, only to be met with the same scenario when they enter secondary school'. Part 2 of Let's join in! Rhymes, Poems and Songs (Martin and Cheater, 1998) is aimed more specifically at older learners in KS2 up to age 13 in KS3. It emphasises the need to revisit many topics and strategies but also to recycle previously taught language in new ways to give learners the sense of moving forward. Although action rhymes are still included at KS2, suggestions for including and developing reading and writing are introduced as a natural extension. In Keep Talking. Teaching in the target language Satchwell (1997) outlines the kind of progression expected at primary level, surprisingly differentiating between 'infants' and 'juniors' rather than key stages or year groups. The ideas contained in Grammar is fun! (Biriotti, 1999) would be extremely helpful both for a specialist and non-specialist in creating motivational activities whilst also planning for progression.

\section{ICT in primary foreign language learning}

Primarily one assumes because of their publication dates and developments which have taken place since then, some volumes in the series make little reference to ICT. There is no reference for example in First steps to reading and writing (Skarbek, 1998) to ICT in the reading section. There are now, of course, PowerPoint versions of stories being used by many primary schools, although the advantages of using ICT are stated in the volume for writing. Equally, because of its age the emphasis in Are you sitting comfortably? Telling stories to young language learners (Tierney and Dobson, 1995) is on the use of flashcards, OHTs and books, whereas in reality in many primary schools now the interactive whiteboard is used for stories. The techniques in these earlier texts, however, are still valid and could be adapted for use with 'big books' or stories using PowerPoint or the interactive whiteboard. It is also true that overusing one resource could result in demotivation, so for language teachers to be exposed to other resources and ideas is of benefit. 
In later volumes, the integration of ICT is more apparent. Symbols for dictionary skills and ICT are used throughout each strand of The Literacy Link (Cheater and Farren, 2001), as the authors make suggestions for developing dictionary skills and cite occasions when ICT can be a helpful tool to support and reinforce learning. Unlike some other Young Pathfinders, this text includes an excellent section on using the internet to develop reading and writing skills encouraging careful and critical consideration of appropriate content, required adaptation, prior knowledge needed and possible follow-up activities. A flying start! Introducing early language learning (de Silva and Satchwell, 1995/2004) also includes a section on the use of ICT which brings the text up-to-date with current practice.

\section{Conclusion}

Taken as a whole, the series provides a wide range of helpful suggestions and methodological advice for specialists and non-specialists alike. There are extensive ideas for planning foreign language learning across KS2 with a host of practical activities for the primary classroom. Throughout the series the emphasis lies in making language learning a positive, motivating learning experience whilst achieving linguistic progression. There are constructive ideas to encourage non-specialists who may lack confidence with the language or who may be concerned about how to fit language learning into the curriculum. Significantly, the series also sees primary language learning within the long-term context of language learning, so that the issue of progression and transition is thoughtfully addressed. Although there are excellent ideas contained within these Young Pathfinder texts, an important challenge for non-specialist teachers is to find the time to read them and to fit them into a coherent, productive programme. It is here that joint collaboration with secondary colleagues or across a local authority to plan and share resources and ideas would be especially beneficial in promoting a successful programme for language learners across both phases. If this is successful, then this should provide learners with the much-needed long term motivation in languages, and work towards reversing the current trend so that pupils will opt to continue with languages at Key Stage 4.

\section{References}

Bevis, R. \& Gregory, A. (2005) Young Pathfinder 13: Mind the gap! Improving transition between Key Stage 2 and 3.

Biriotti, L. (1999) Young Pathfinder 8: Grammar is fun

Cheater, C. \& Farren, A.(2001) Young Pathfinder 9: The literacy link

Datta, M. \& Pomphrey, C. (2004) Young Pathfinder 10: A world of languages. Developing children's love of languages. 
de Silva, J. \& Satchwell $(1995,2004)$ Young Pathfinder 1: A flying start! Introducing early language learning

Driscoll P., Jones J. and Macrory, G. (2004) The provision of foreign language learning for pupils at Key Stage 2. Research report. DfES.

Martin, C. (1995) Young Pathfinder 2: Games and fun activities

Martin, C. \& Cheater,C. (1998) Young Pathfinder 6: Let's join in! Rhymes, poems and songs.

Martin, C. \& Farren, A. (2004) Young Pathfinder 12: Working together. Native speaker assistants in the primary school.

Muijs, D., Barnes, A., Hunt, M., Powell, B., Martin, C., Arweck, E. Lindsay, G. (2005) Evaluation of the Key Stage 2 language learning pathfinders. London, DfES, available at http://www.dfes.gov.uk/research/data/uploadfiles/RR692.pdf.

OFSTED (2002) Changing schools - an evaluation of the effectiveness of transfer arrangements at age 11. Available at http://www.ofsted.gov.uk/publications/docs/309.pdf, (accessed 10.3.06).

Satchwell,P.(1997) Young Pathfinder 4: Keep talking. Teaching in the target language.

Skarbek, C. (1998) Young Pathfinder 5: First steps to reading and writing.

Tierney,D. \& Dobson,P. (1995) Young Pathfinder 3: Are you sitting comfortably? Telling stories to young language learners.

Tierney,D. \& Hope, M. (1998) Young Pathfinder 7:: Making the link. Relating languages to other work in the school. 